\title{
Projekte für die Weiterbildung: An Ideen fehlt es nicht
}

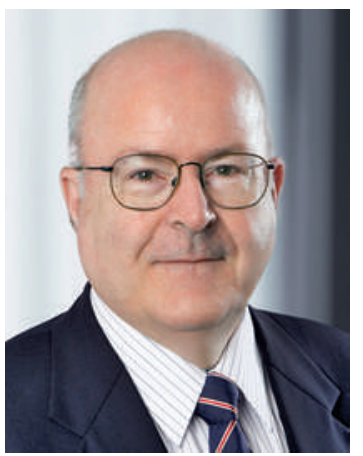

Am Anfang standen eine Sorge und eine Idee. Die Sorge betraf die Rahmenbedingungen für die ärztliche Weiterbildung, wie wir sie heute in den Spitälern erleben: Bei Visitationen und anderen Kontakten mit Weiterbildungsverantwortlichen stossen wir immer wieder auf Unzufriedenheit, Irritation und sogar Resignation. Zwar sind die Begeisterung und die Verpflichtung, ärztliches Wissen und Können an die kommende Generation weiterzuvermitteln, durchaus nicht erloschen. An vielen Weiterbildungsstätten aber nagen die Realitäten eines hektischen Spitalalltags, der Mangel an personellen und finanziellen Ressourcen sowie die administrativen Belastungen des Kaders massiv an dieser Begeisterung. Viele verantwortliche Ärztinnen und Ärzte haben das Gefühl, sie müssten sich die Zeit und Energie für das aktive Vermitteln von Kompetenzen irgendwie zusammenklauben und unter den heute so dominanten ökonomischen Aspekten rechne sich die Weiterbildung nicht mehr.

\section{Die Weiterbildungsverantwort-}

\section{lichen stehen zunehmend unter}

\section{Zeit- und damit auch Kostendruck.}

Das darf doch nicht sein! Beim Konzipieren und Evaluieren aller möglichen Massnahmen zur Unterstützung des «teaching» tauchte die Idee auf, eine Projektausschreibung zu lancieren. Das Budget erlaubte es glücklicherweise, hunderttausend Franken für die Förderung von Projekten zu reservieren, die zum Ziel haben, die Weiterbildenden zu unterstützen oder zu entlasten. Im Oktober 2013 erfolgte die Ausschreibung, die mit Absicht keine enge Definition der erwarteten Projekte beinhaltete, sondern zum Einreichen von Anträgen verschiedenster Inhalte ermunterte. Wir warteten gespannt.

$\mathrm{Zu}$ unserer Freude war die Resonanz überraschend gross: 62 Anträge wurden eingereicht. In ihrer Mehrzahl waren sie von ausgezeichneter Qualität und zum Teil wirklich originell. Natürlich gab es auch einzelne «Schlaumeier-Projekte», die einfach zum Ziel hatten, einen finanziellen Beitrag für die Alltagsweiterbildung zu mobilisieren - die innovativen und kreativen Konzepte überwogen aber bei weitem. Inhaltlich reichte das Spektrum von «e-learning tools» und hybriden Methoden über Simulationsprojekte, «skill lab»-Entwicklungen, Aufbau von Datenbanken, Evaluations-Apps für den klinischen Alltag, standardisierten Kursen zur Einführung am Arbeitsplatz, «teach the teacher»-Angeboten bis hin zu Workshop-Drehbüchern. Die Jury stand vor der erfreulichen, aber nicht einfachen Aufgabe, aus einer ganzen Serie von för-

\section{Die Resonanz auf die SIWF-Projekt- förderung war mit 62 eingereichten Weiterbildungsanträgen überwältigend.}

derungswürdigen Projekten eine Auswahl zu treffen, die dem finanziellen Spielraum entsprach. Eines der massgebenden Kriterien war, dass das Projekt später auch ausserhalb des Entwicklungsortes genutzt werden kann. Die Juroren bewältigten ihre Aufgabe, die zu einer eigentlichen Qual der Wahl wurde, im Rahmen zweier Bewertungsdurchgänge. Schliesslich wurden vier Projekte ausgewählt, die im Rahmen dieser Ausschreibungs-Premiere unterstützt werden. Deren Ziele sind der Aufbau einer Radiologie-Lernplattform, das aktive Erarbeiten einer Zytopathologie-Datenbank durch die Ärzte in Weiterbildung, die Entwicklung eines Trainingssystems in minimalinvasiver Chirurgie und das Erarbeiten eines Simulationskurses in mechanischer Beatmung für die Intensivmedizin.

Und nun? Nun sind wir zuversichtlich, dass die ausgewählten Projekte erfolgreich realisiert werden können und auch längerfristig und über ihren Entstehungsort hinaus einen Beitrag zur Weiterbildung leisten. Und nun wissen wir, dass an vielen Orten gute Ideen zur Weiterbildung schlummern, die mit einer solchen Ausschreibung geweckt und von denen im Rahmen unserer Möglichkeiten einige auf den Weg zur Realisierung gebracht werden können. Eine zweite Auflage folgt bestimmt.

Dr. med. Werner Bauer, Präsident des Schweizerischen Instituts für ärztliche Weiter- und Fortbildung SIWF 